\title{
RECENT RESULTS PERTAINING TO THE HELIUM-RICH STARS
}

\author{
N. R. WALBORN \\ Cerro Tololo Inter-American Observatory, La Serena, Chile
}

\begin{abstract}
The term helium-rich stars here refers to those stars with apparent spectral types near B2 and helium lines which are enhanced, but not stronger than the hydrogen lines. The previously known helium-rich star HD 184927 has been found to be a spectrum variable; the equivalent widths of the helium lines changed by an average of $46 \%$ between two observations. HD 186205 has been newly recognized as a member of the helium-rich class. Further work toward understanding the rapidly variable $\mathrm{H} \alpha$ emission in the prototype helium-rich star, Sigma Orionis E, is in progress in co-operation with Dr J. E. Hesser at CTIO.
\end{abstract}

\section{DISCUSSION}

Garrison: I have spectra of stars in the small tight cluster which includes the multiple star $\sigma$ Ori. The cluster forms an extremely narrow main sequence and contains a 'He weak' star in addition to $\sigma$ Ori $E$, the 'He rich' star. The 'He weak' star is a mild one with a B5 colour and a B8 spectral type.

Morgan: On the assumption that $\sigma$ Ori $\mathrm{E}$ is a member of the Orion association, do you find that it has normal luminosity for a B2V star?

Walborn: The presence of $\sigma$ Ori $\mathrm{E}$ in a multiple system which contains an earlier-type mainsequence star, as well as a normal B2V star with nearly identical apparent magnitu te and colour to $E$, places strong constraints of interpretations of the helium-rich star.

Bell: Is there any evidence for the existence of $\mathrm{He}^{3}$ in these stars?

Walborn: No.

Schatzman (To Dr Nissen and Dr Walborn): Mrs S. Vauclair has a nice explanation of the difference between He poor and He rich stars, as a result of competition between diffusion and stellar wind.

Nissen: Yes, I agree that the explanation by S. Vauclair is very interesting. .

Note added in proof. A periodicity of 1.19 has been found in the spectrum, light, and color variations of Sigma Orionis E. References: Publ. Astron. Soc. Pacific 87, 613, 1975; Astrophys. J. Letters, April 15, 1976. 